\title{
Correlative Light Electron Microscopy with High Time Resolution and Ultrastructural Preservation
}

Paul Verkade

\author{
Max Planck Institute of Molecular Cell Biology and Genetics, Electron Microscopy Technology \\ Development, Pfotenhauerstrasse 108, Dresden 01307, Germany.
}

With the evolution of better live cell imaging techniques and equipment there has also been an increasing interest / effort to develop techniques and tools to translate these light microscopical data to electron microscopy (so-called Correlative Light Electron Microscopy, CLEM). Important issues to solve are the preservation of ultrastructure, time resolution, and detection of fluorescent markers (most importantly GFP). Whereas most existing techniques either compromise with ultrastructural preservation by using chemical fixation others using fast freezing techniques need at least 30 seconds to transfer their sample from imaging at the light microscope until it is frozen. Cellular events that only happen rarely or are very fast, such as budding from the Golgi/TGN or intracellular membrane transport, are thereby missed.

To circumvent these compromises and problems a new tool is presented that allows us to view a sample live under the light microscope and rapidly freeze this same sample within 5 seconds by means of high pressure freezing. This new tool makes it possible to study those intracellular events that are either fast or only occur occasionally (see Figure 1).

To develop this tool / instrument we modified an EMPACT high-pressure freezer (Leica Microsystems) by adding a Rapid Transfer System (RTS). The sample (in this case cultured cells grown on sapphire disc) is first viewed in the light microscope in a pre-prepared holder and then transferred to the high-pressure freezer. The pre-prepared holder consists of a modified EMPACT specimen carrier with a bigger opening for light microscopical viewing, the sapphire disc with cells, and a custom-made finder grid for re-localization of a particular cell of interest (see Figure 2). In more detail, the sample is viewed live in the light microscope where first of all DIC / Phase and fluorescent overview images are taken with the finder grid in the field of view in order to re-locate the cell of interest. Then the image is zoomed in on this cell of interest and only the fluorescent channel needs to be imaged live (see also Figure 1). When a structure or event of interest is observed the sample holder is manually transferred to the RTS (1 to 2 seconds). The RTS transfers the sample holder automatically to the EMPACT, where it is frozen. This second step is performed within 3 seconds. This gives a total time resolution of under 5 seconds. After freezing, the samples are processed for freeze substitution, where extraction of the water and fixation is done at low temperature. They are then further processed by conventional methods of resin infiltration so sections can be viewed in the electron microscope (see also below). Using this set up we reached good freezing results in over $90 \%$ of samples such as PtK2 (see figure 3) and MDCK cells.

At the moment the focus is on the detection of the fluorescent label (GFP and also mRFP) for electron microscopy. A comparison is made between different methods. These include; preembedment immunogold labeling after rehydration of the samples; post-embedment labeling of Lowicryl embedded material and photo-conversion of the fluorophore. 

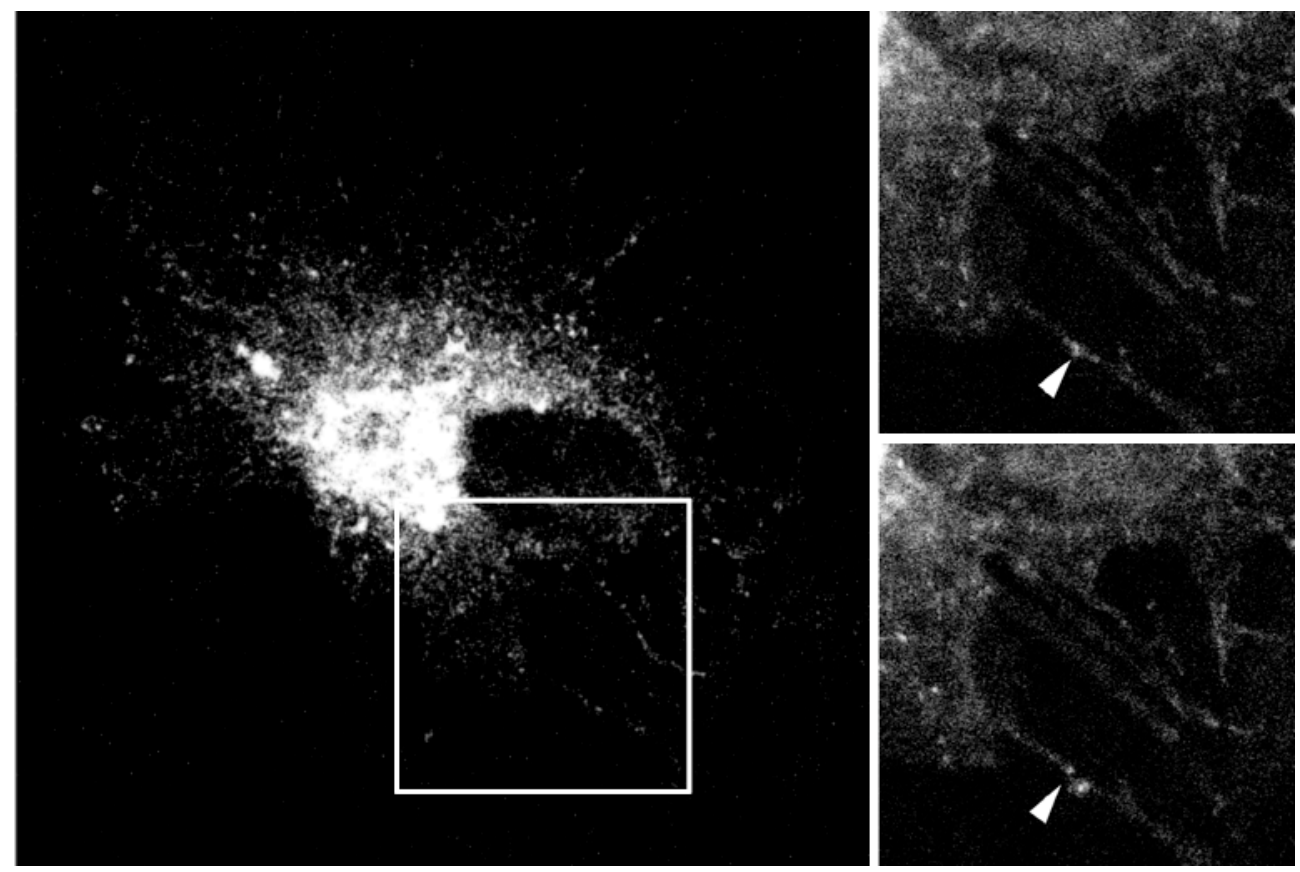

Figure 1: Fluorescence micrographs of a MDCK cell expressing VSVG-GFP. After release of a TGN block transport carriers containing VSVG-GFP leave the Golgi on their way to the plasma membrane. The enlargements show "live" images taken 10 seconds after each other. In the top panel there is only 1 transport carrier, in the bottom there are 2 .

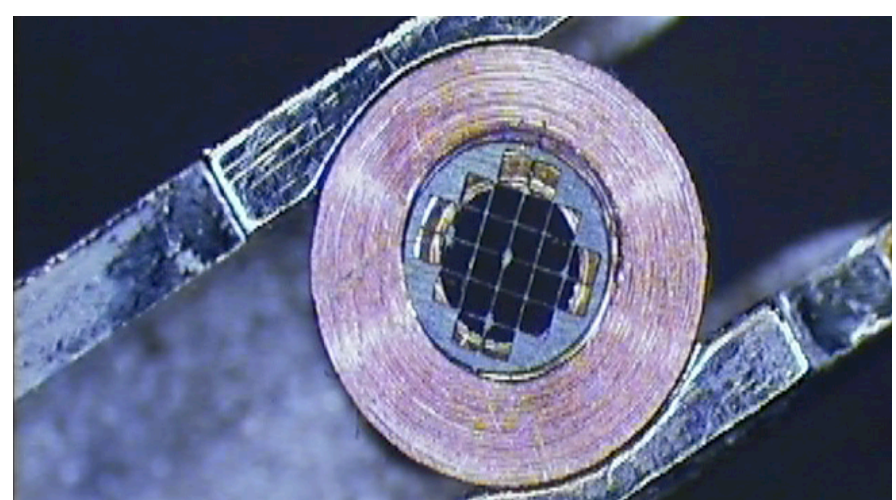

Figure 2: The sample holder of the Rapid Transfer System. The opening of the standard copper carrier has been enlarged to do phase contrast or DIC. In the carrier is a sapphire disc with cells and on top is a finder grid for re-localization.

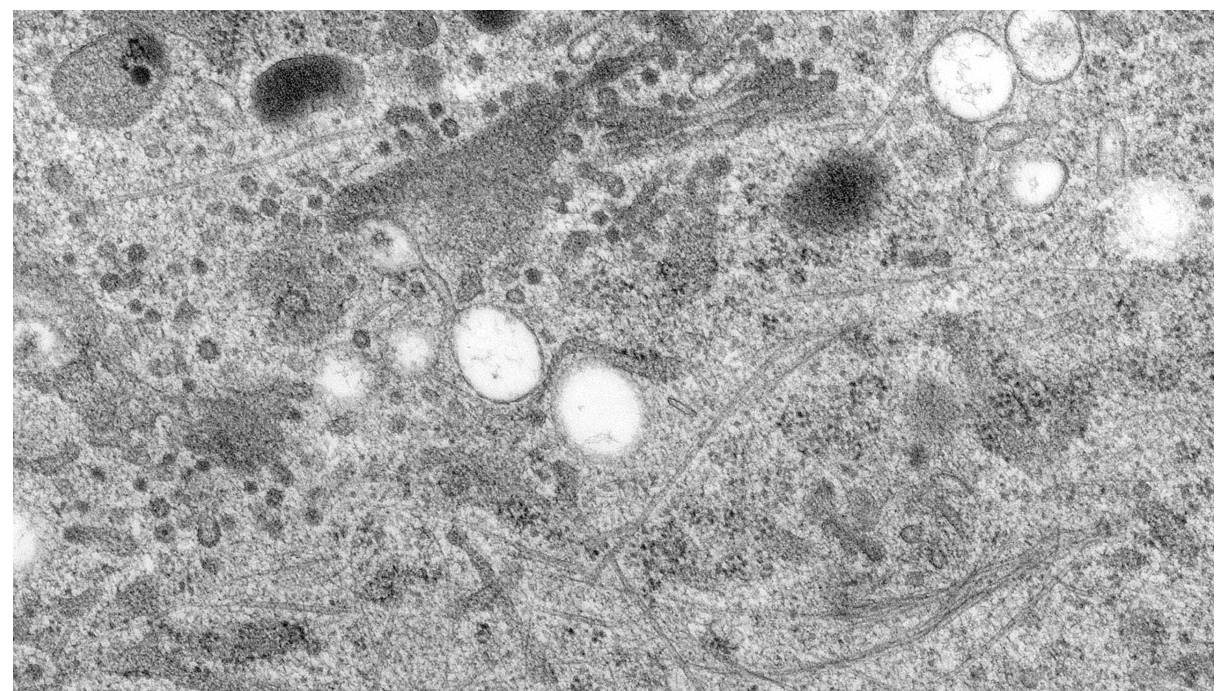

Figure 3: Electron micrograph of the Golgi area of a PtK2 cell. The sample was high pressure frozen with the Rapid Transfer System, freeze substituted and afterwards embedded in Epon. 